\title{
Starfire Optical Range 3.5-m telescope adaptive optical system
}

James M. Spinhirne, Jeff G. Allen, George A. Ameer, James M. Brown, Julian C. Christou, et al.

James M. Spinhirne, Jeff G. Allen, George A. Ameer, James M. Brown, Julian C. Christou, Terry S. Duncan, R. J. Eager, Mark A. Ealey, Brent L. Ellerbroek, Robert Q. Fugate, Gary W. Jones, R. M. Kuhns, David J. Lee, William H. Lowrey, Michael D. Oliker, Raymond E. Ruane, David W. Swindle, Joshua K. Voas, Walter J. Wild, Keith B. Wilson, John L. Wynia, "Starfire Optical Range 3.5-m telescope adaptive optical system," Proc. SPIE 3353, Adaptive Optical System Technologies, (11 September 1998); doi: 10.1117/12.321689 


\title{
The Starfire Optical Range 3.5m Telescope Adaptive Optical System
}

\author{
J.M. Spinhirne ${ }^{a, 1}$, J.G. Allen ${ }^{b}$, G.A. Ameer ${ }^{a}$, J.M. Brown II ${ }^{c}$ J.C. Christou' ${ }^{c}$ T.S. Duncan ${ }^{c}$, R.J. Eager ${ }^{a}$, \\ M.A. Ealey ${ }^{d}$, B.L. Ellerbroek ${ }^{c}$, R.Q. Fugate ${ }^{c}$, G.W. Jones ${ }^{a}$, R.M. Kuhns ${ }^{c}$, D.J. Lee ${ }^{c}$, W.H. Lowrey ${ }^{a}$, \\ M.D. Oliker ${ }^{b}$, R.E.Ruane ${ }^{a}$, D.W. Swindle ${ }^{b}$, J.K. Voas ${ }^{c}$, W.J. Wild, K.B. Wilson ${ }^{b}$, and J.L. Wynia ${ }^{a}$ \\ ${ }^{2}$ Boeing North American, PO Box 5670, Kirtland AFB, NM 87185 \\ bAdaptive Optics Associates, 54 Cambridge Park Dr., Cambridge, MA 02140 \\ 'Starfire Optical Range, USAF Research Laboratory, 3550 Aberdeen Ave. SE, Kirtland AFB, NM 87117

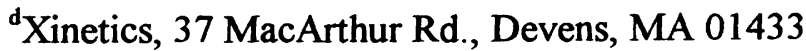 \\ 'University of Chicago, Department of Astronomy \& Astrophysics, \\ 5640 S. Ellis Ave., Chicago, IL 60637
}

\begin{abstract}
A 941 channel, 1500 Hertz frame rate adaptive optical (AO) system has been installed and tested in the coudé path of the $3.5 \mathrm{~m}$ telescope at the USAF Research Laboratory Starfire Optical Range. This paper describes the design and measured performance of the principal components comprising this system and presents sample results from the first closed-loop tests of the system on stars and an artificial source simulator.
\end{abstract}

Keywords: Starfire Optical Range, adaptive optics, deformable mirror, atmospheric compensation

\section{INTRODUCTION}

A principal goal of the Starfire Optical Range (SOR) adaptive optics (AO) program is the development and demonstration of a high order, high bandwidth AO system for use with the SOR $3.5 \mathrm{~m}$ telescope. A 941 channel, $1500 \mathrm{Hertz}$ frame rate natural guide star AO system has now been implemented and tested. The design and measured performance of the major components of the system are described in this paper. A second paper in this volume ${ }^{1}$ quantifies the current performance of the system in terms of compensated star images and tip/tilt jitter measurements.

The key optical and electronic components of the $3.5 \mathrm{~m}$ AO system are illustrated schematically in Figure 1 . The $3.5 \mathrm{~m}$ telescope gathers the incident light and reflects it down the coudé path. A K mirror is used to maintain a fixed image orientation independent of the pointing direction of the telescope. Relay optics are used to reimage the primary mirror onto the deformable and steering mirrors with the proper pupil sizes. These mirrors are actively controlled to compensate for tip/tilt and higher order wave-front aberrations using measurements of the residual closed-loop errors provided by the Shack-Hartmann wave-front sensor and the track error sensor. Two visible/near infrared scoring cameras are used to record images for post processing.

The electronic components of the AO control system include a gradient processor which calculates in real time the wavefront slope errors from the wave-front sensor camera data. The wave-front reconstructor next generates the optical phase most consistent with the measured gradients. This calculation is implemented as a matrix multiply. These phase values are the measured error signals which are then processed by the control filter and applied to the deformable mirror by the high voltage amplifiers. The track processor receives the track error from one of several sensors and provides control signals to

${ }^{1}$ Corresponding author: Email:spinhirne@qmailer.plk.af.mil; Telephone: (505) 846-6217; Fax: (505) 846-2213 


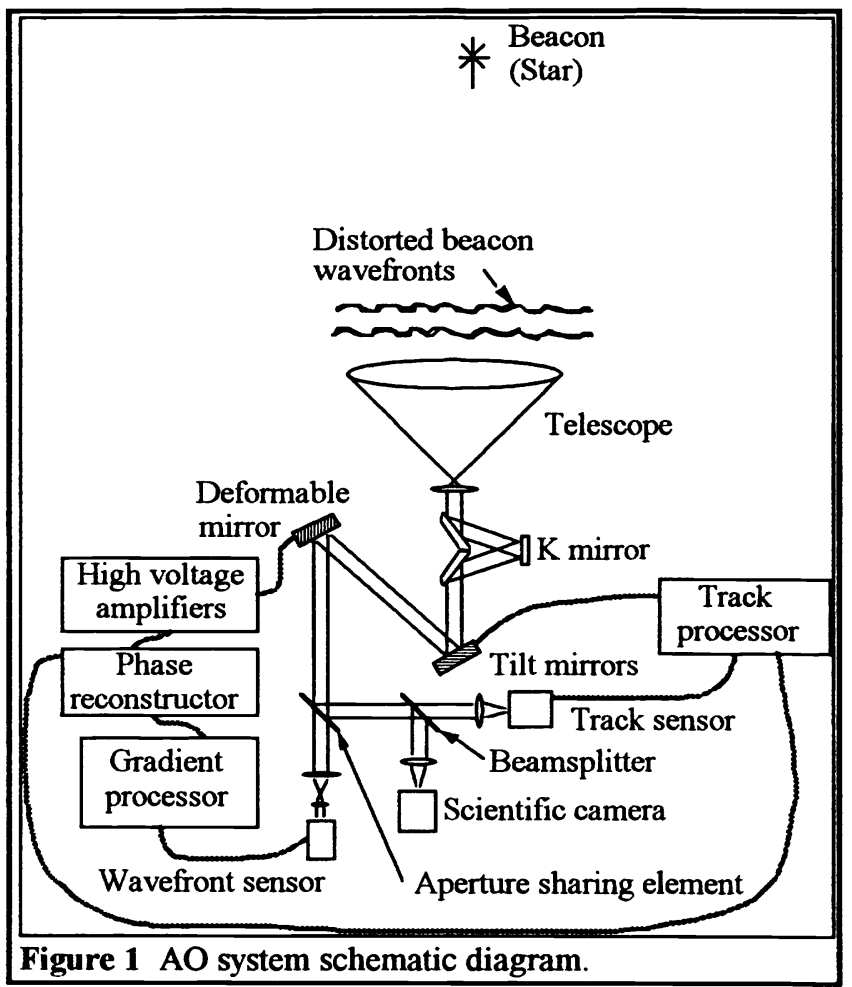

the fine and coarse steering mirrors. Scoring sensor images, wave-front sensor gradient measurements, and reconstructed phase values are acquired by separate computer interfaces for post-processing.

The AO system aperture geometry is illustrated in Figure 2, which summarizes the geometric relationships between the telescope aperture, the actuator locations on the deformable mirror, the subapertures of the Shack-Hartmann wave-front sensor as defined by the lenslet array, and the pixels of the wave-front sensor CCD camera. The deformable mirror is located at a pupil plane whose diameter is $21 \mathrm{~cm}$, which corresponds to $\mathbf{3 0}$ actuator spacings. The lenslet array is also located at a pupil plane which has the proper magnification so that the image of each actuator centerline can be located at the intersection of four lenslets. Each lenslet produces a Shack-Hartmann spot. This array of spots is imaged onto the CCD camera of the wave-front sensor so that the spot separation is exactly four pixels. The center quad of each $4 \mathrm{x}$ 4 pixel group is used for measurement of the spot location.

The remainder of the paper describes the design and performance of the above components in greater detail. The $3.5 \mathrm{~m}$ telescope and relay optics are reviewed in section 2 , and the wave-front and scoring sensor optics are described in section 3. Section 4 summarizes wave-front sensor performance. The AO control electronics are discussed in

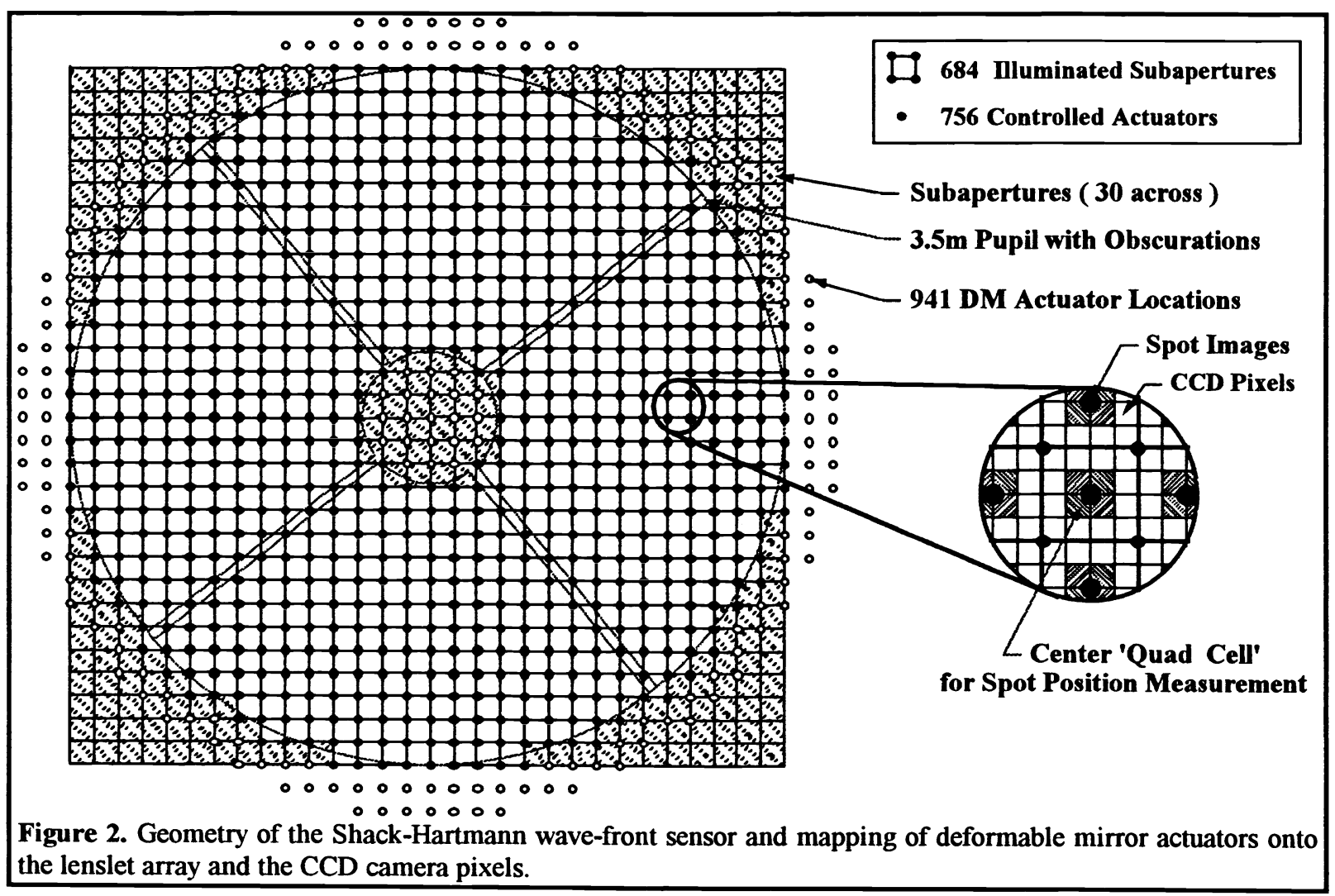


section 5, and the control algorithms implemented by these electronics are described in section 6 . Section 7 outlines the track sensors and processors used for tip/tilt compensation. Sample first results from the AO system are presented in Section 8. Additional results and associated system performance estimates are described in the paper by Lee and Ellerbroek to be found later in this volume. The design and performance of the deformable mirror will be described in a separate paper currently in preparation.

\section{TELESCOPE AND RELAY OPTICS}

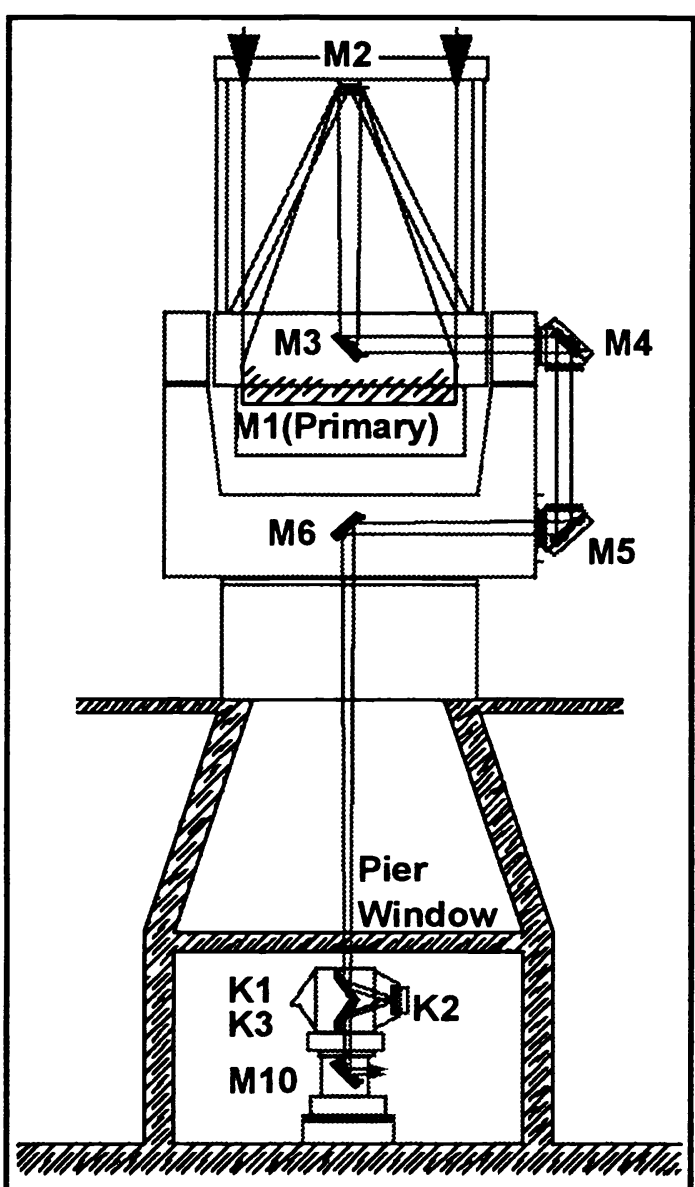

Figure 3. SOR 3.5 meter telescope and coudé path.
The Starfire Optical Range 3.5m telescope proper has been described in a previous paper. ${ }^{2}$ The telescope is of the open frame type with a truss structure supporting the secondary spider. The dome is retractable, allowing the telescope to be completely exposed to the surrounding atmosphere.

Figure 3 shows the $3.5 \mathrm{~m}$ telescope and its coude path. The Cassegrain telescope consists of an $F / 1.5$ parabolic primary mirror and a hyperbolic secondary mirror. The magnification is 10 and the back focal distance is 31.5 meters. The unvignetted field of view (FOV) is one milliradian. An image de-rotation $\mathrm{K}$-mirror is installed in the vertical path at the ground floor level. Above the K-mirror, a fused silica window provides separation of the temperature stabilized laboratory area (below the window) from the beam path above which is at ambient temperature during telescope use. A fold flat (M10) below the K-mirror rotates to select one of several optical paths.

The coudé room optical relay system, illustrated in Figure 4, begins with mirror $\mathrm{M10}$ at the base of the telescope pier. A range of requirements, many associated with the use of adaptive optics, are key to understanding the approach taken in the design of the optical relay. The design must be all reflective to maximize through-put and spectral bandwidth, and must provide pupils for the steering mirror, the deformable mirror, and sensor interfaces. The steering mirror must be placed at a "small" (under $12 \mathrm{~cm}$ diameter) pupil. Placement at a pupil is required so that no pupil motion results from tilt correction, thus assuring a stable exit pupil at the wave-front sensor. A "small" pupil is required so that fast tilt correction can be achieved with current technology steering mirrors. The deformable mirror must be placed at a $21 \mathrm{~cm}$ diameter pupil to match the prespecified actuator geometry. A small (under $80 \mathrm{~mm}$ diameter) exit pupil must be provided for interface to the sensors. This will allow lenses and dichroic beam splitters to be smaller, easier to fabricate, and less costly.

The optical train must allow laser propagation for beams focused at ranges from 10 kilometers to infinity. No element should be at a laser beam focus. All foci should be slower than F/50 to minimize air breakdown effects. Finally, the physical layout of the optical relay must be configured to fit into the available laboratory space. Further discussion on the design and performance of the coudé room optical relay may be found in an earlier paper. ${ }^{3}$

\section{SENSOR OPTICS}

The sensor optical system is illustrated in Figure 5 and consists of all the components down beam of the toric T6. These components begin with an IR long wave pass dichroic beam splitter with an edge wavelength near one micrometer. This optic reflects all light of wavelengths for which silicon detectors work well. The transmitted IR light will be sensed using 


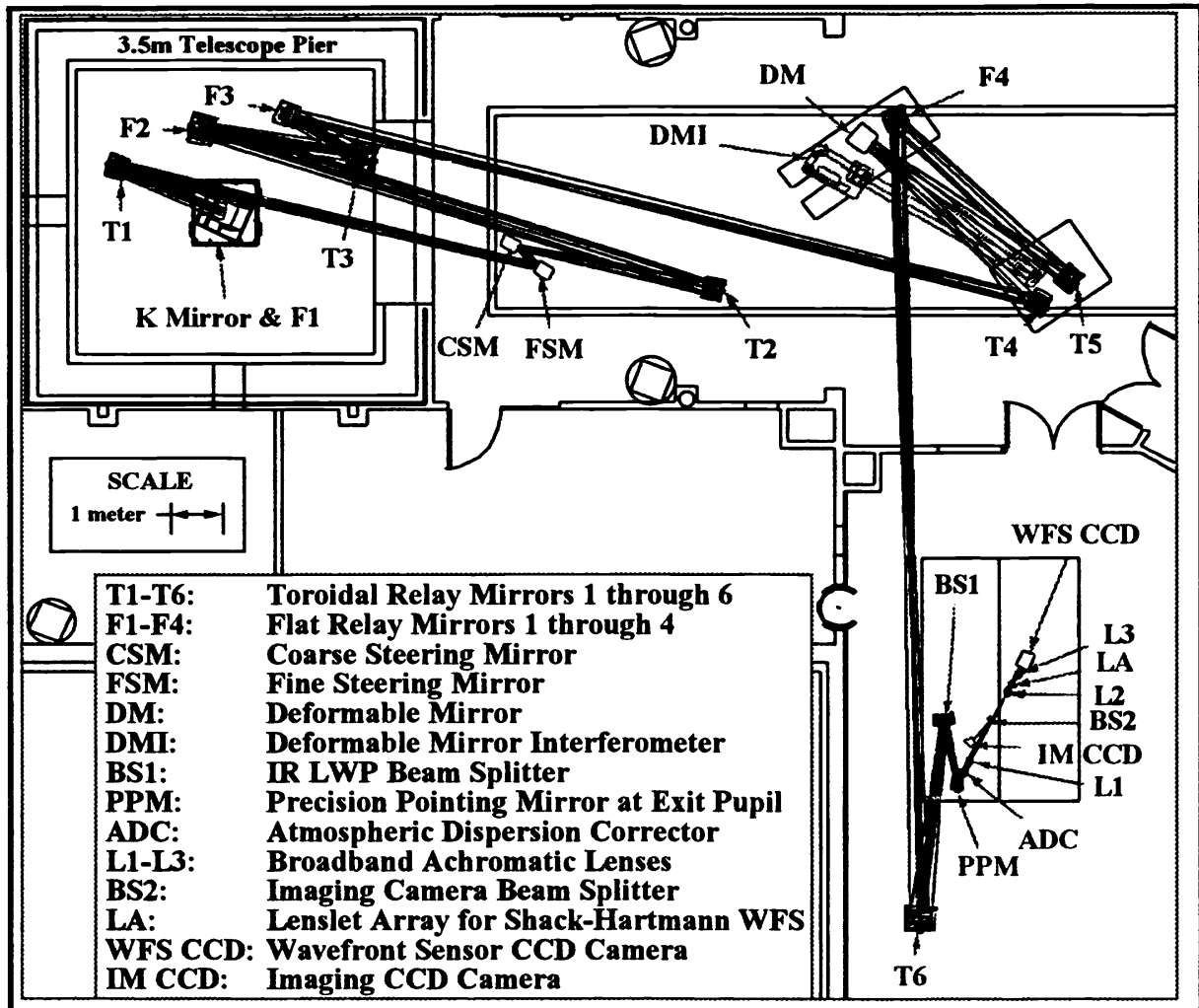

Figure 4. Coudé room optical relay. other detectors. The reflected light is now sufficiently limited in spectral band that refractive optics can be used in the remainder of the path.

A precision pointing mirror (PPM) is located at a $7.25 \mathrm{~cm}$ diameter pupil. The mirror is remotely adjustable and can place any object within 150 microradians (30 arc $\mathrm{sec})$ of the center of the telescope field of view on the optical axis of the sensors. The relatively small 20 degree angle of incidence and the location of the mirror at a pupil assure minimal coupling of mirror motion to pupil motion at the wave-front sensor.

Following the PPM is the atmospheric dispersion corrector (ADC) which compensates for the dispersion of the atmosphere for zenith angles to 70 degrees over the spectral band of 0.47 to 1.03 micrometers. It is located as near the pupil as is practicable to minimize pupil blurring at the

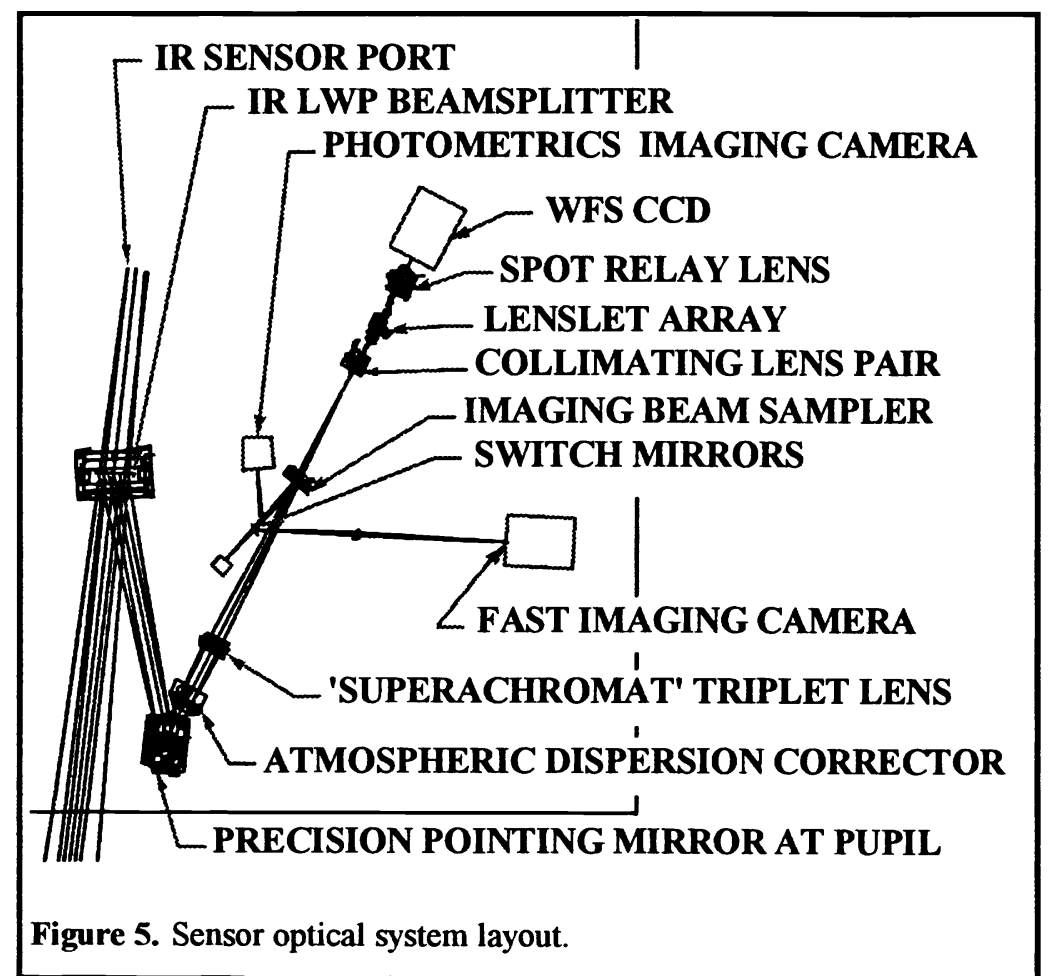
wave-front sensor. The 'superachromat' triplet lens produces an $F / 20$ focus that provides for Nyquist rate sampling of the beam using a Kodak 2048 by 2048 pixel CCD camera having 9 micrometer square pixels.

The imaging beam sampler provides light to several imaging cameras. One is the Kodak CCD camera for long exposure images. Another is a low noise, fast framing CCD camera for characterization of system jitter and short exposure imagery. Pixel size is set to 120 nanoradians, the Nyquist rate for a 0.85 micron wavelength. Several different beam splitters, ranging from broadband partial reflectors to narrow spectral band high reflectors are available for use as the imaging beam sampler. The beam splitter selection is based upon the imaging requirements for a particular experiment. The transmitted light is used for wave-front sensing. This configuration allows for great flexibility in the spectrum used for imaging while providing all of the remaining light to the wave-front sensor for improved performance with relatively dim objects. 
The collimating lens pair consists of back-to-back doublets that collimate the output beam and generate an exit pupil at the Shack-Hartmann sensor lenslet array. The lenslet array consists of square lenslets, each having a side dimension of 200 micrometers and a focal length of 6.3 millimeters. Thirty lenslets span the exit pupil diameter. The separation between the two doublets in the collimating lens is adjusted to fine tune the exit pupil diameter to match the width of each lenslet to the deformable mirror interactuator spacing. Finally, the wave-front sensor spot relay lens images the Shack-Hartmann spots onto the wave-front sensor CCD camera with the proper magnification to place one spot every four pixels.

\section{WAVEFRONT SENSOR PERFORMANCE}

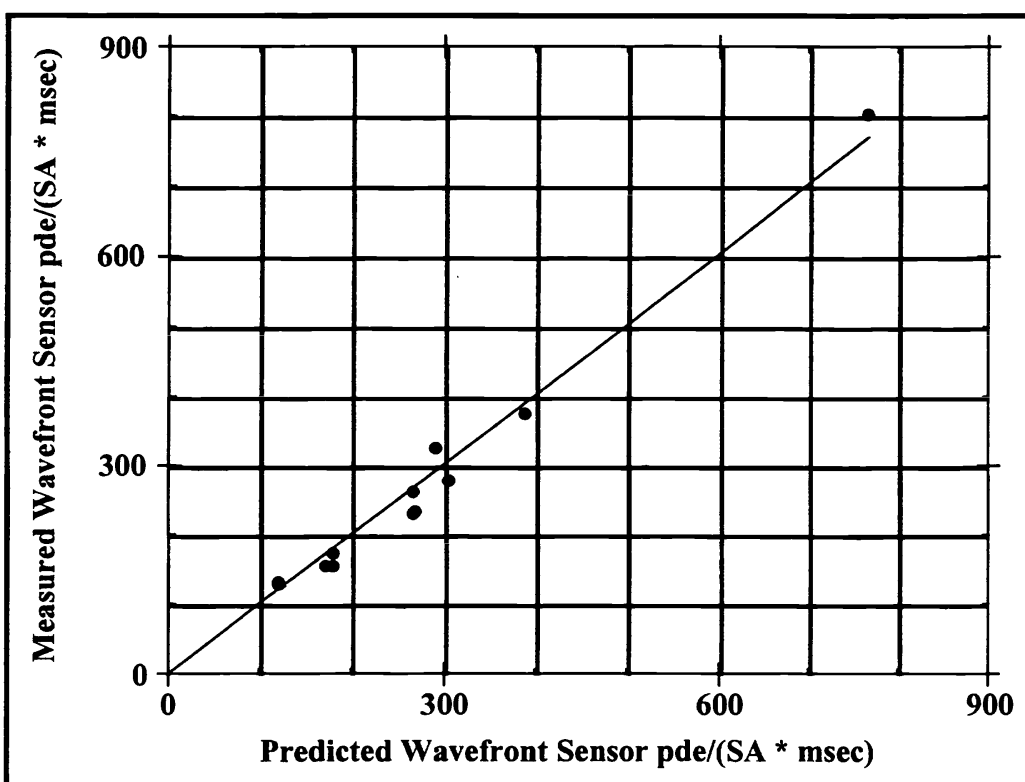

Figure 6. Wave-front sensor radiometry measurements.

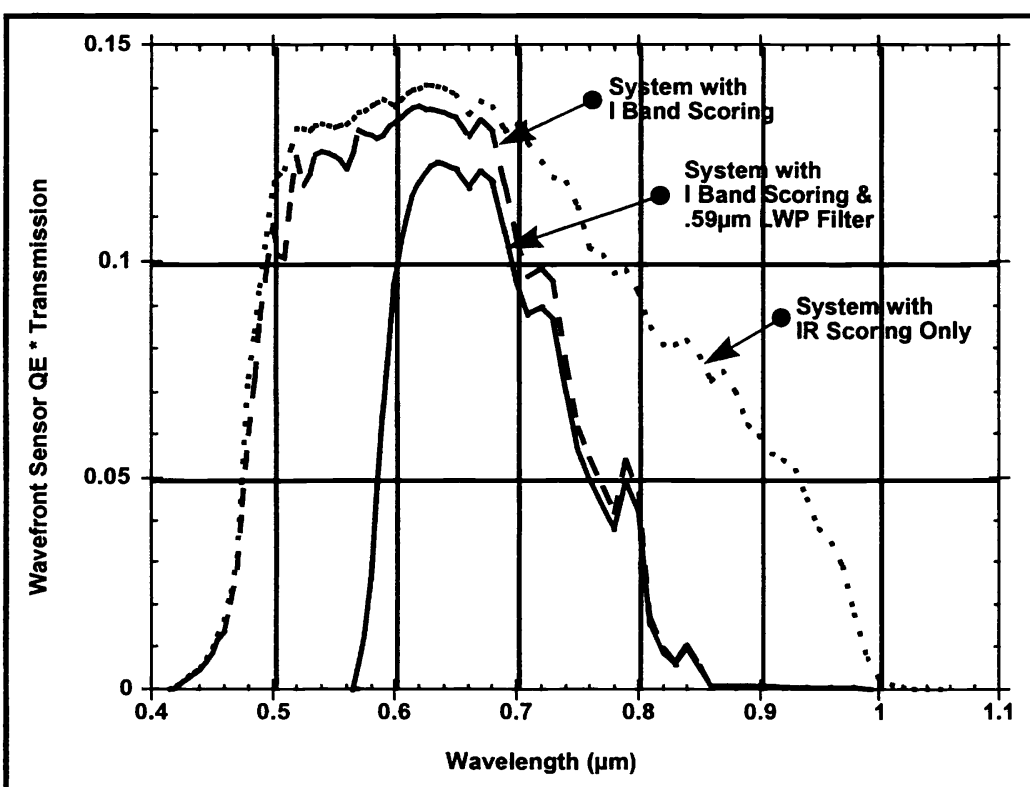

Figure 7. Wave-front sensor spectral sensitivity.
The first-order parameters determining wavefront sensor tilt measurement accuracy include end-to-end optical system transmittance, detector quantum efficiency, detector read noise, and the slope of the transfer curve relating input wave-front tilt to the Shack-Hartmann spot motion measured by the wave-front sensor. Radiometric measurements for the wave-front sensor were recorded for several stars and were used to anchor the spectral response model for the system. Figure 6 shows the fit to the expected signal levels for several stars of varying magnitude and color.

The spectral sensitivity estimates derived from the previous data are illustrated in Figure 7. The lowest line is the current spectral transmission for the system set for I band scoring measurements. The short wavelength cut-off at 0.59 microns is currently required because of chromatic aberrations (lateral color) in the wave-front sensor spot relay lens system. Once this problem is corrected, the spectral transmission will become as shown by the middle curve. The top curve plots the maximum signal available when the entire visible spectrum is used for wave-front sensing. In this case, scoring measurements would be performed in the infrared. Figure 8 plots wave-front sensor signal level as a function of stellar magnitude based upon the data presented in Figures 6 and 7. The spectral bands are as shown in the previous graph. The operational limit of 50 photodetection events per wave-front sensor subaperture at a $500 \mathrm{Hertz}$ sampling rate is based upon measured performance. ${ }^{1}$ The effect of measurement noise upon system performance appears to be negligible at signal levels greater than about 200 photodetection events per subaperture at 1500 Hertz. 


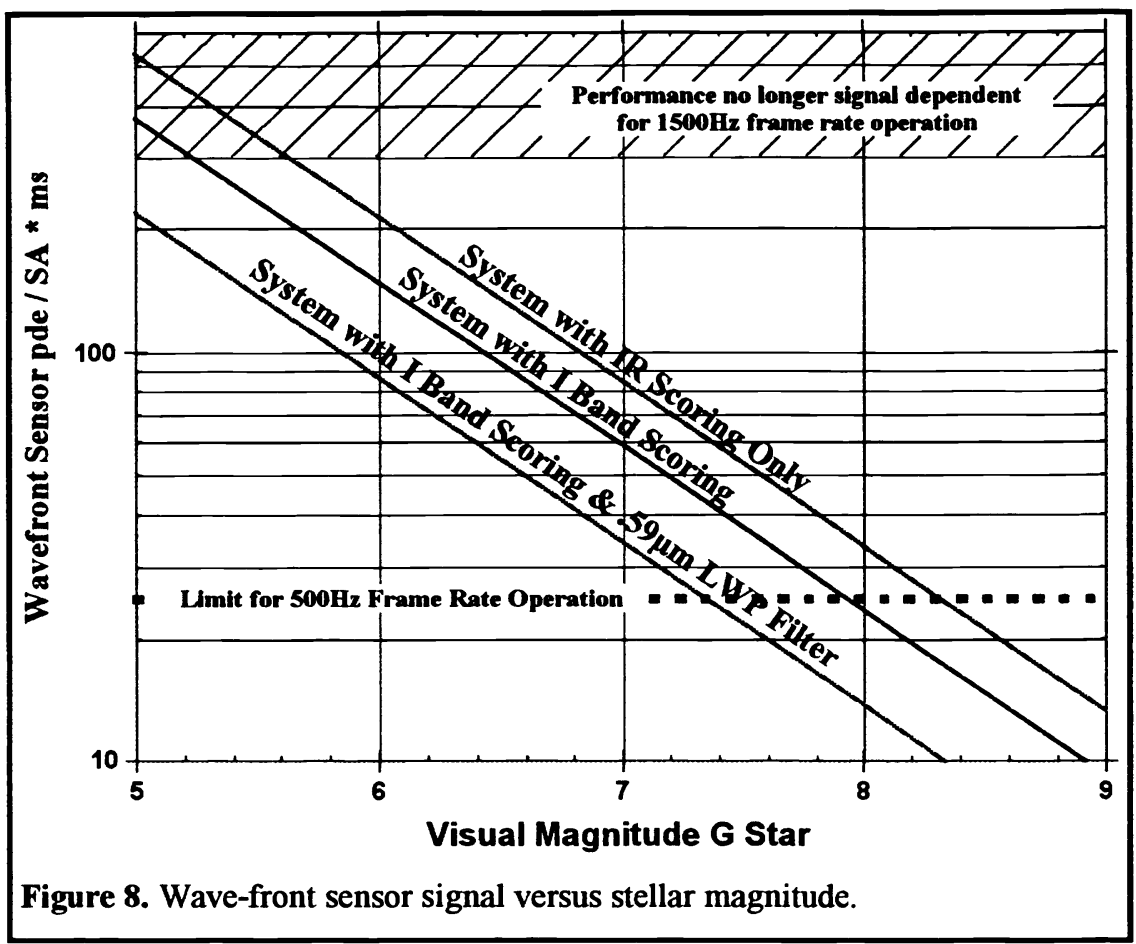

Shack-Hartmann spot locations are estimated from the intensities measured by a 2 by 2 array of CCD pixels using the standard tilt measurement expression for a quadrant detector as illustrated in Figure 9. Offsets are subtracted from each pixel to account for detector biases, and the relative imbalances of the calibrated intensities are treated as $X$ and $Y$ spot positions errors which are proportional to the wave-front gradients over the subaperture. A reference gradient is subtracted from each measurement to remove errors due to uncommon path aberrations and imperfections in the lenslet array. Using a local broadband source, the output gradients from the wave-front sensor were measured as a function of horizontal ( $\mathrm{X}$ axis) spot translation. The translations were calibrated and are expressed in terms of micro-radians of tilt at the input aperture of the telescope.

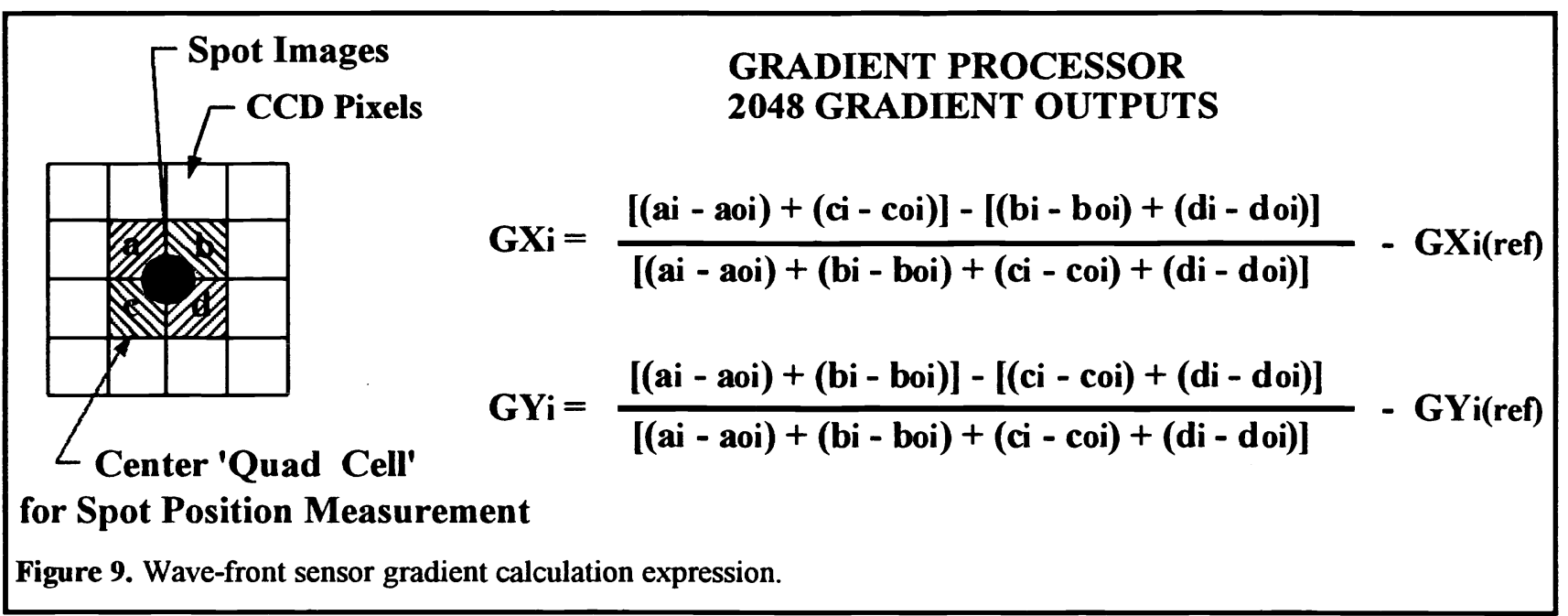

The gray band in Figure 10 is the envelope of all 812 measurements superimposed on one another. The centered line is the mean of the measured response curves, and the steeper curve is the response expected from an identically sized perfect quad cell illuminated over the same spectral band by a perfect (diffraction limited) optical system. There is an RMS zero crossing slope variation of approximately $10 \%$ and the zero imbalance point has an RMS variation of 0.37 microradians, or about 43 nanometers of optical path difference across a subaperture.

The slope of the mean transfer curve is $\mathbf{5 7 \%}$ of the theoretical maximum. Two factors are known to contribute to this degradation of the measured performance. First, the pixels in the CCD camera do not have perfectly sharp edges. This blurring of the pixel boundary was determined to produce a slope reduction to $75 \%$ of ideal for a similar WFS used at the $\mathrm{SOR}^{4}{ }^{4}$ The CCD camera used for the $3.5 \mathrm{~m}$ wave-front sensor is of similar design, but the pixel blurring has not yet been measured. Second, the optical train to the WFS is not perfect and will introduce some (primarily chromatic) image blurring. 


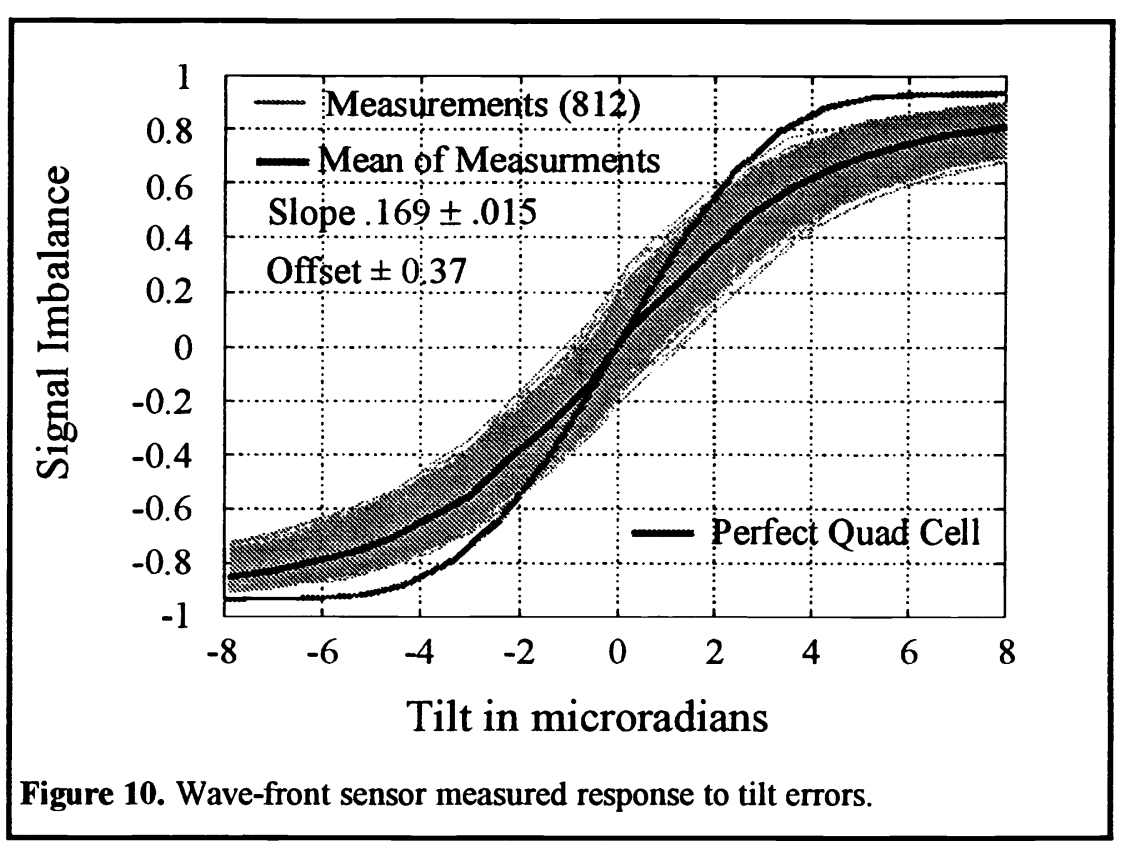

wave-front sensor system illustrating how the quadrant detector algorithm outlined in Figure 9 is implemented in hardware. The wave-front sensor CCD array is read out through 4 analog outputs, each with a rate of 4.5 mega-pixels/second. The usual mode of operation is to discard two rows of pixels between the rows of quad cell pixels since these data are not used in the calculations and the read-out time can be decreased to reduce overall processing latency. Four digitizers are located in the camera housing which, with the 2:1 Mux, provide 2 digital output data streams. Because of the distance from the WFS camera to the processor, the digital data is transmitted over optical fiber. At the processor, four image and control processor

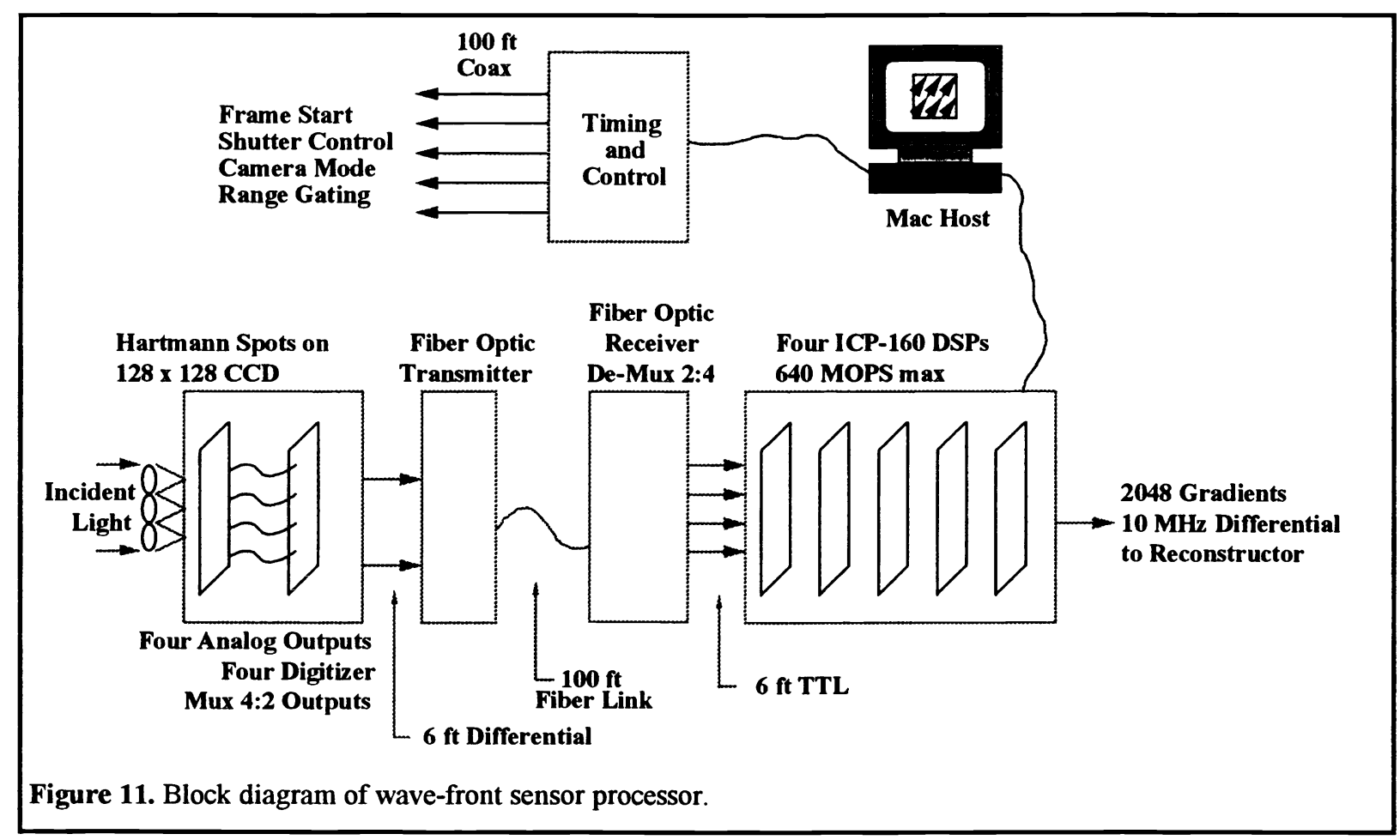


(ICP) DSP's (an Adaptive Optics Associates custom chip) perform the quad cell processing algorithm and output the 2048 gradient measurements to the reconstructor. Data acquisition, control of various timing signals, and some specialized data processing such as that required during alignment of the wave-front sensor are all implemented in the host Apple Macintosh computer.

Figure 12 illustrates the principal components of the wave-front reconstructor. The input data arrives as a single data stream of 2048 gradients which is received by the input module. This module duplicates the data and sends a copy to each of eight engine modules. Each engine module consists of 128 separate DSP's with one assigned to each deformable mirror actuator or auxiliary output channel. The engine module provides the matrix multiply function for phase reconstruction from the gradient measurements and also applies the control filter to define the DC error rejection and bandwidth of the control loop. Parallel outputs from each module transfer the phase command information to the inputs of the High Voltage Amplifiers.

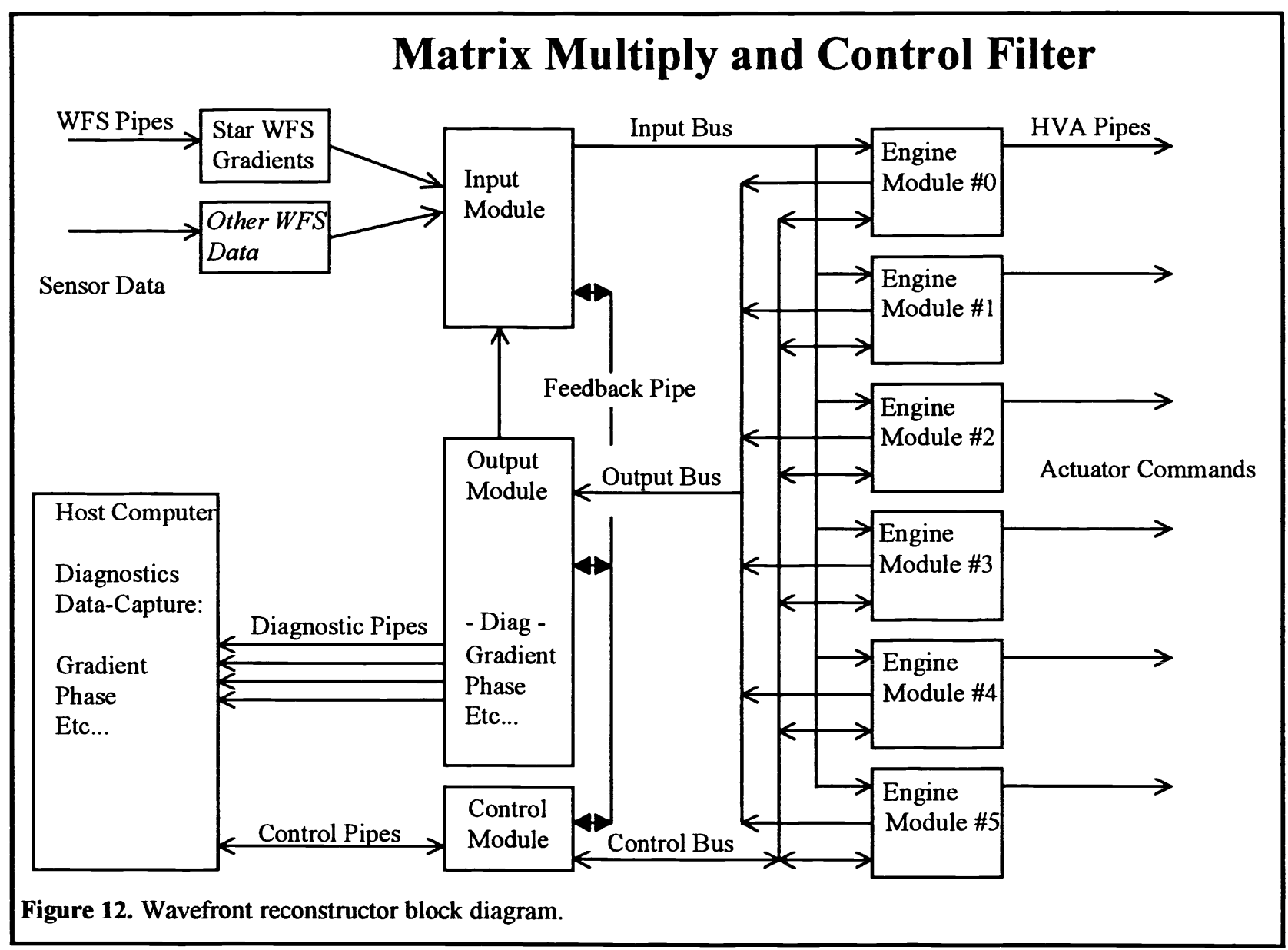

Data acquisition from the reconstructor is accomplished by an output module which collects and formats data from each of the engine modules. The module can also pass data back to the Input Module for feedback of parameters such as full aperture tilt or piston so that an integrator control filter might be used without build-up of modes not sensed by the wavefront sensor. The host computer provides a means to capture up to 64,000 contiguous frames of phase data or an equivalent amount of synchronized phase and gradient data. It is also used to specify the algorithms used in the engine modules, including the choice of reconstruction matrix coefficients and the control filter constants. 


\section{ADAPTIVE OPTICS CONTROL ALGORITHM}

The adaptive optics control algorithm is specified by a set of reconstruction matrix coefficients and the control filter constants. All reconstruction matrices used to date have been derived using the classical least-squares solution ${ }^{5}$ for the socalled Fried actuator/subaperture geometry as illustrated in Figure 2. The effects of sensor and actuator gain variations and residual misalignments between the wave-front sensor and deformable mirror have been neglected. The free parameters in the design of the reconstruction algorithm include (i) the choice of which wave-front sensor subapertures to use in estimating the phase, and (ii) the so-called "slaving algorithm" to use for edge actuators not adjacent to such subapertures. Full aperture tilt is removed from the reconstructed wave-front because these two modes are separately sensed and compensated by the system's tilt sensor and steering mirrors.

Best results to date have been obtained with a reconstruction matrix derived for a set of subapertures which yields 688 actively controlled actuators and 3 rings of slaves. The slaved actuators are driven to minimize a weighted sum of squares of command differences between all pairs of adjacent actuators containing at least one slave. This is a least squares criterion which may be minimized via a matrix multiply. Although the pupil diameter at the deformable mirror would seem to correspond more closely to 756 active actuators and 2 rings of slaves, reconstruction matrices derived for this aperture geometry do not yield stable results except at unacceptably low control loop bandwidths. We suspect that this difficulty may be associated with wave-front sensor gain variations in partially illuminated edge subapertures. A reconstruction matrix derived for a compromise aperture geometry with 728 active actuators has yielded encouraging results with the local source

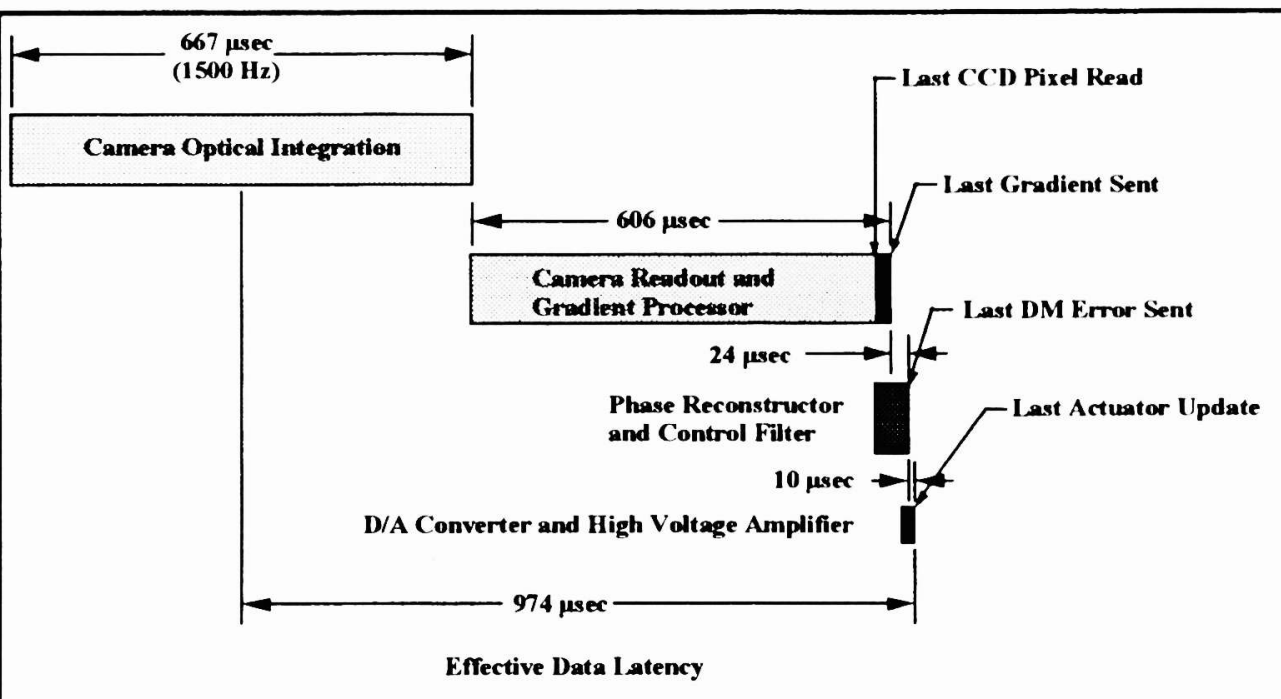

Figure 13. Data latency in AO system processing. simulator but has not yet been tested against stars.

The temporal error rejection and phase margin for the $\mathrm{AO}$ system control loop is determined by the choice of control filter coefficients. The response of the deformable mirror actuators is fast relative to the wave-front sensor sampling rate, so that evaluation and optimization of the filter coefficients depends primary upon the latency of the control loop. The data latency for the AO system is illustrated in Figure 13. The first block of the timeline is the optical integration period of the wavefront sensor camera, which

introduces an average data latency equal to one half of the integration period. The second block is the wave-front sensor camera readout and gradient processing. Camera readout dominates this delay with the last gradient output occurring 24 microseconds after the last pixel is read. The phase reconstructor adds an additional 24 microseconds of delay, and the high voltage amplifier system introduces an additional 10 microseconds of delay. This gives a total processing delay of 640 microseconds and an effective data latency of 974 microseconds. This latency is one of the fundamental parameters governing loop bandwidth capability and was used in the development of models to predict loop performance.

To validate these loop performance models, the error rejection of a single actuator was measured by adding a discreetly sampled sine wave error to the digital data at the point just after the control filter was applied. The resulting residual error was applied to the deformable mirror while the loop was closed using two different loop gains (and therefore loop bandwidths). The results obtained with a wave-front sensor frame rate of $1500 \mathrm{Hertz}$ are given in Figure 14, which plots the residual error as a function of frequency. The lines indicate the predictions generated by the model of the loop and the 
discrete points are the measured values. Increasing the loop gain increases bandwidth and improves the DC error rejection as expected. The loop bandwidth defined by the 0.7 error rejection point is slightly over $50 \mathrm{Hertz}$ for the higher of the two loop gains. This is the loop bandwidth used during much of the system testing. Analogous test data were recorded for a wave-front sensor frame rate of $833 \mathrm{Hertz}$. At this frame rate, the loop bandwidth is only slightly decreased because loop performance is determined primarily by gain and latency values and the latency is dominated by the camera readout rate.

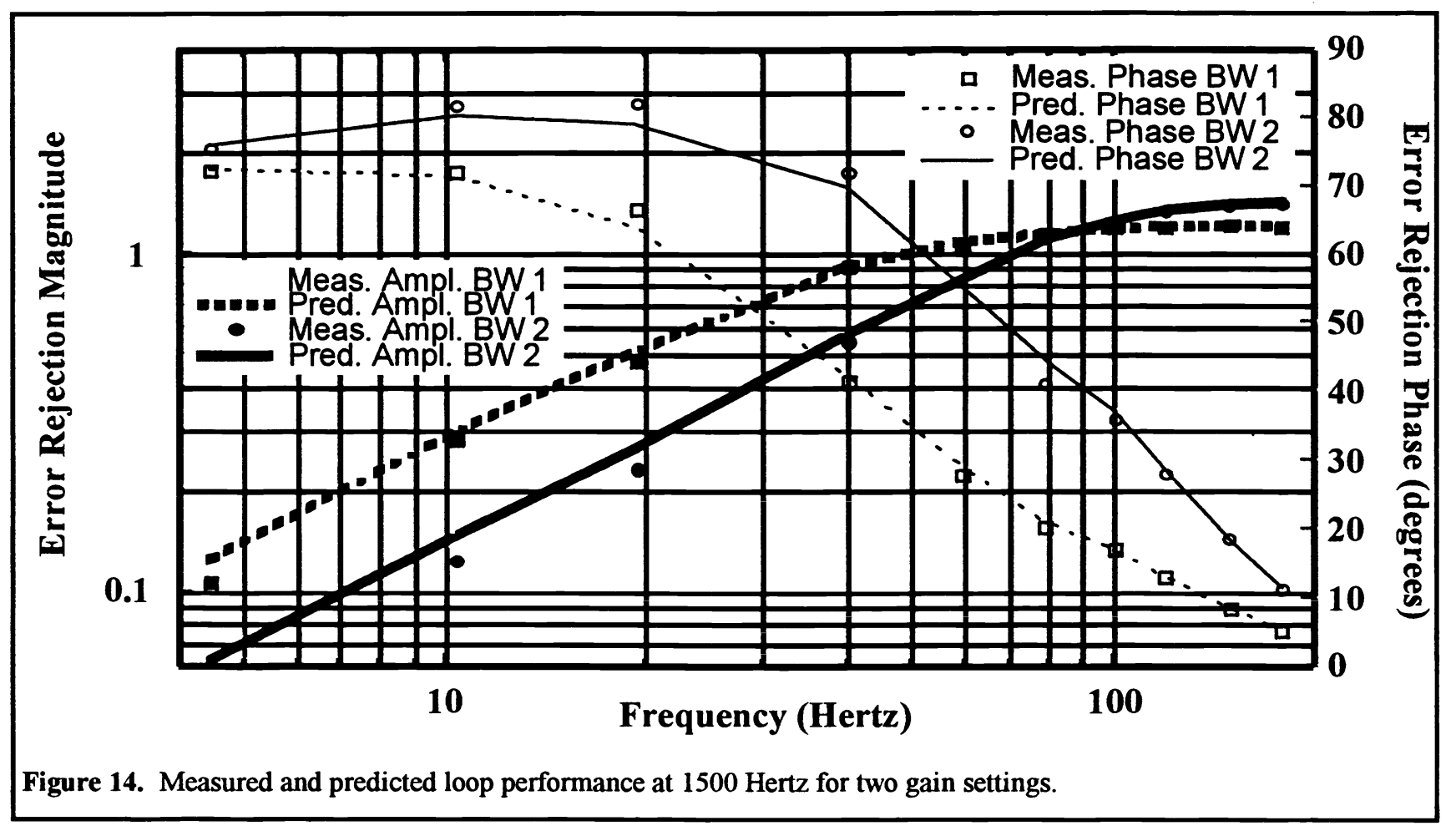

The AO system processing hardware is capable of implementing higher-order control filters, not just the simple low pass filter now in use. A modeling effort is underway to compensate for the effects of latency by optimizing the control filter coefficients. Faster wave-front sensor cameras planned for the system will also significantly reduce the $\mathbf{5 8 0}$ microseconds of latency due to readout.

\section{TRACKING SYSTEM}

Figure 15 is a block diagram of the tracking system implemented at the $3.5 \mathrm{~m}$ telescope. Tip and tilt corrections are accomplished using two steering mirrors. The fine steering mirror (FSM) has a quick response but a very limited throw, while the coarse mirror (CSM) has much greater dynamic range but is limited in bandwidth to about 10 Hertz. The tracking control law is implemented to allow the FSM to compensate for disturbances above 5 Hertz while the CSM handles the lower frequencies. The two movable mirrors work in a woofer-tweeter configuration, analogous to audio speakers.

The control theory is implemented in software by a Texas Instrument DS1003 digital signal processor (DSP). The DSP hardware is built by dSPACE GmbH. The control law is designed and tested with MatLab and Simulink. A graphical user interface is accomplished using Cockpit, a software program offered by dSPACE, to communicate with the DSP while it is executing the tracker software. 


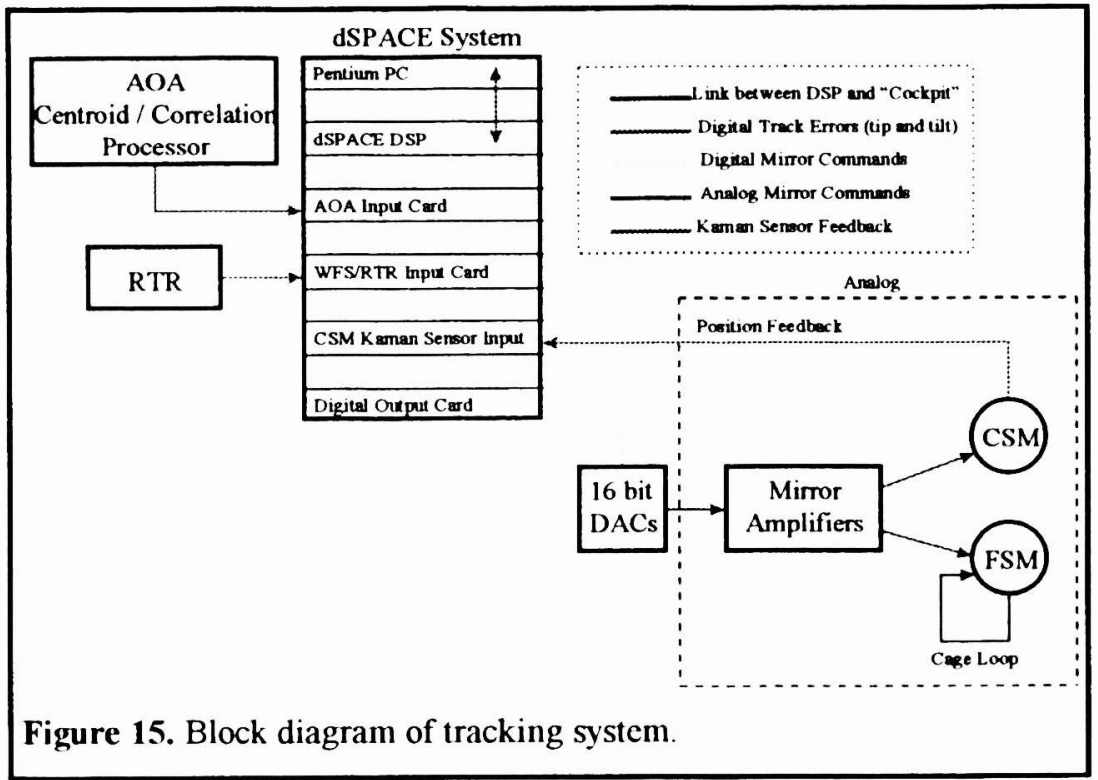

The FSM has a built-in cage loop which compensates for hysteresis of the piezoelectric actuators, allowing control of this mirror without position feedback. The CSM does not have a built-in cage loop, and position measurements from three Kaman sensors are digitized with 12 bit precision and input into dSPACE. Once the Kaman sensors are digitized, software compiled from the Simulink design performs the function of the CSM cage loop.

dSPACE receives track error signals from one of the two track sensors currently in use at the 3.5 meter telescope. These errors consist of tip and tilt values which dSPACE uses to calculate mirror commands at a 10 kilo-Herty rate. Both currently available sensors run at much lower sampling rates and provide new data asynchronously to dSPACE. Input prototype boards capture the data and signal the DSP when new data is present. The DSP checks for new data every time through the 10 kilo-Hertz loop and implements the control law with new data, if present, and using the latest data until new data arrives. The filtered commands are digitally sent to 16 bit DACs through another dSPACE prototyping board. The voltage is sent to amplifiers which then drive the mirrors

The 3.5 meter telescope's first operational track sensor was the wave-front sensor/real time reconstructor tracker (WFS/RTR tracker), which uses the average tilt data from the wave-front sensor. In late February 1998, a centroid/correlation track processor was moved from the 1.5 meter telescope and became operational. This tracker, developed by Adaptive Optics Associates (AOA), uses the $64 \times 64$ visible/NIR CCD scoring camera built by AOA and MIT/Lincoln Labs. Since the camera data is sent to both the scoring computer and the AOA track processor, the data rate for this tracker is determined by the

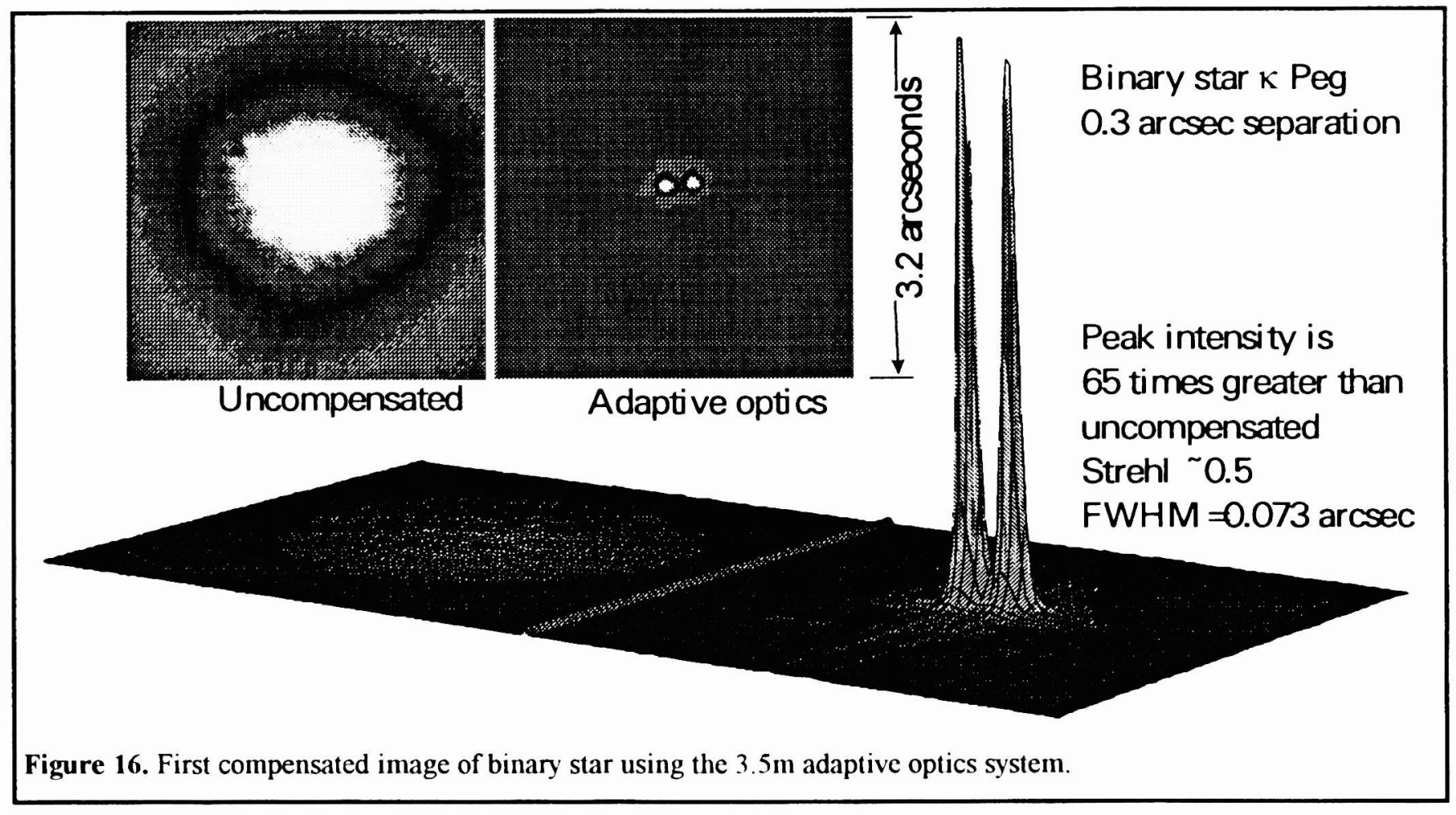


operator of the scoring camera and is usually 1818 Hertz. The AOA processor uses the camera data to determine tip and tilt errors using three modes of operation: acquisition, centroid, or correlation. Acquisition mode calculates the centroid of the image after a low pass filter while centroid mode calculates the centroid of the instantaneous image. The correlation mode is used to calculate the position of a resolved object with sharp contrasts.

\section{SAMPLE FIRST RESULTS}

Two binary star images illustrating the present performance of the SOR $3.5 \mathrm{~m}$ telescope AO system are presented in Figures 16 and 17. See the paper by Lee and Ellerbroek' later in this volume for a more extensive and quantitative description of system performance.

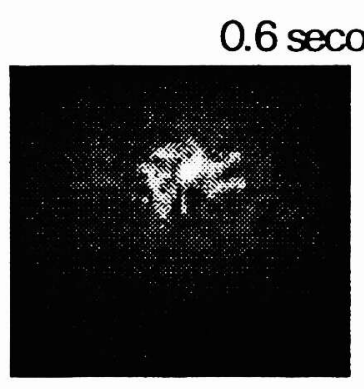

Uncompensated

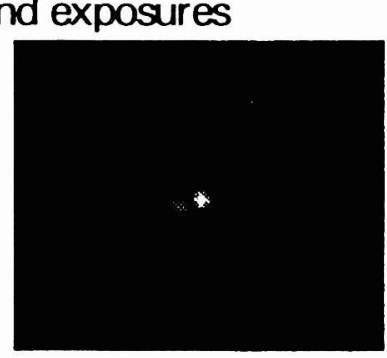

Adaptive optics
Binary star 104 Tau

0.098 arcsec separation

$\mathrm{FWHM}=0.059$ $\operatorname{arcsec}$

$(\lambda / D=0.050)$

Strehl ratio 0.5 Peak intensity is 71 times greater in the compensated image

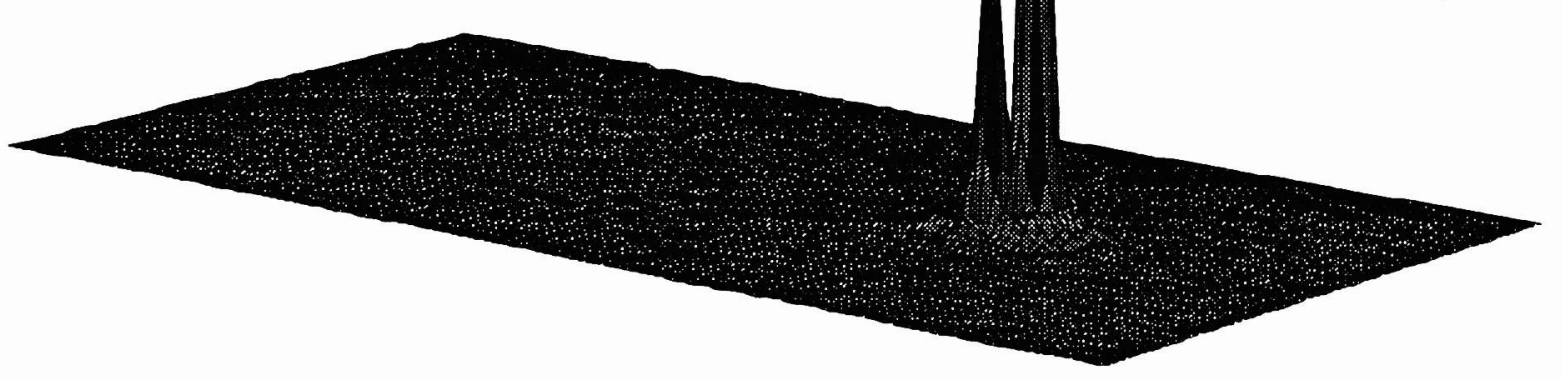

Figure 17. Compensated image showing resolution of 0.1 arc second binary.

\section{REFERENCES}

1. D.J. Lee and B.L. Ellerbroek, "First results for the Starfire Optical Range 3.5m telescope adaptive optics system," this volume.

2. Fugate, et al., 'First Observations with the Starfire Optical range 3.5 Meter Telescope," Symposium on Astronomical Telescopes and Instrumentation for the $21^{\text {st }}$ Century, SPIE Proc., 1994.

3. J. M. Spinhirne and G. A. Ameer, "Adaptive optics using the $3.5 \mathrm{~m}$ Starfire Optical Range Telescope," Adaptive Optics and Applications, R.K. Tyson and R.Q. Fugate editors, SPIE Proc., Vol. 3126, pp. 257-268, 1997.

4. TL Pennington, D.W. Swindle, M.D. Oliker, B.L. Ellerbroek, and J.M. Spinhirne, "Performance measurements of Generation III wavefront sensors at the Starfire Optical Range," Adaptive Optical Systems and Applications, R.K. Tyson and R.Q. Fugate editors, SPIE Proc., Vol. 2534, pp. 327-337, 1995.

5. J. Herrmann, "Least-squares wave-front errors of minimum norm," J. Opt. Soc. Am. 70, 28-35 (1980). 